\title{
Program Administrator Burnout in Graduate Medical Education: a Longitudinal Study
}

\author{
Alana M. Ewen, MPH'(D), Natalie Gittus, JD², Mikhail C.S.S. Higgins, MD, MPH \\ Sandra Palma, BA $A^{4}$, Kathryn Whitley, BA, C-TAGME ${ }^{5}$, and Jeffrey I. Schneider, MD ${ }^{1,6} \odot$
}

'Graduate Medical Education, Boston Medical Center, Boston, MA, USA; ${ }^{2}$ Medical Education, Baylor University Medical Center, Dallas, TX, USA; ${ }^{3}$ Division of Interventional Radiology, Department of Radiology, Boston University School of Medicine, Boston Medical Center, Boston, MA, USA; ${ }^{4}$ Department of Radiology, Brigham and Women's Hospital, Boston, MA, USA; ${ }^{5}$ Department of Family Medicine, Boston University School of Medicine, Boston Medical Center, Boston, MA, USA; ${ }^{6}$ Department of Emergency Medicine, Boston University School of Medicine, Boston, MA, USA.

BACKGROUND: Little is known about the level of burnout among program administrators (PAs) in medical education and its impact on the trainee environment.

OBJECTIVE: To investigate variations in burnout levels over a 1-year period among a national cohort of PAs and examine any associations between perceived support and isolation.

DESIGN: A 1-year longitudinal study conducted to assess burnout levels among PAs across the USA. The Copenhagen Burnout Inventory (score range, 0-100) was used to measure burnout over one academic year (July 2017June 2018). The generalized estimating equations model was used to measure changes in burnout levels from the start of the academic year. To explore the differences in burnout scores across question response levels, a oneway ANOVA test was utilized and reported as least squares means $\pm \mathrm{SD}$.

PARTICIPANTS: Individuals who self-identified as PAs in a graduate medical education training program. Among the 1084 persons nationwide who expressed interest, 904 (83\%) completed the baseline survey; 29 of the 42 (69\%) local administrators completed the survey. "Clients" defined as interns, residents/fellows, and medical students. MAIN MEASURES: Change in burnout score using the validated tool. Hypothesis formulated prior to data collection.

KEY RESULTS: Among the 931 participants, the 3rd quarter (March 2018) marked the lowest average personal burnout score (change from the start of academic year, 3.67; $p<0.001,95 \% \mathrm{CI}-5.77$ to -1.58 ) and work-related burnout score (change, $-3.03 ; p<0.001,95 \% \mathrm{CI}-5.01$ to - 1.06). Client-related burnout was the lowest in September 2017 (change, $-1.46 ; p=0.491 ; 95 \%$ CI -3.54 to 0.62). June 2018: those who strongly agreed to feeling isolated in their current position had an increased personal $(69.1 \pm 18.4 \mathrm{SD})$, work-related $(72.5 \pm 20.8 \mathrm{SD})$, and client-related (42.3 $\pm 23.7 \mathrm{SD})$ burnout score.

CONCLUSIONS: PA burnout levels fluctuate over the academic year and are shown to increase as feelings of isolation grow.

Electronic supplementary material The online version of this article (https://doi.org/10.1007/s11606-020-05853-8) contains supplementary material which is available to authorized users.

Received January 8, 2020

Accepted April 13, 2020

Published online May 12, 2020
J Gen Intern Med 35(11):3248-53

DOI: $10.1007 / \mathrm{s} 11606-020-05853-8$

(C) Society of General Internal Medicine 2020

\section{INTRODUCTION}

Burnout is a syndrome defined by three composite elements - emotional exhaustion, depersonalization, and decreased personal accomplishment. ${ }^{1}$ While the pervasiveness of burnout among physicians in training and physicians in general is well established (more than $50 \%$ in some studies), ${ }^{2}$ there has been relatively little work done to understand the impact of burnout on residency/fellowship program administrators $(\mathrm{PAs}){ }^{3,4}$

The tendency for burnout to function as a contagion, infectively spreading through members of a given health care team, has been well documented. ${ }^{5,}$ In recent years, many have suggested new approaches aimed at altering the trajectory of the physician burnout crisis while identifying mechanisms to shift the culture in medicine to account for increasing dissatisfaction and poorer quality of life among members of the medical team. ${ }^{7-11}$ However, notable gaps in the literature still persist, and larger scale, longitudinal studies investigating changes in PAs' burnout remain scarce in the literature.

Importantly, the Accreditation Council for Graduate Medical Education (ACGME) has begun to acknowledge the psychological strain felt by administrative staff (PAs) within Graduate Medical Education (GME) and the important role these staff play in combating trainee burnout, as evidenced by the creation of the Coordinator Advisory Group. ${ }^{8}$ In the spring of 2016, the Coordinator Advisory Group, which serves as advisors to the ACGME, was established to meet the needs of residency/fellowship coordinators. Since its inauguration, group members have developed a toolkit focused on coordinator well-being and have outlined the role coordinators play in the conversations around resident well-being. ${ }^{12}$

PAs are at the core of the learning environment, supporting the perennial educational needs of residents and fellows. They are intrinsically involved in the administrative processes for 
medical residency/fellowship training programs that have become increasingly complex. As important members of the medical education team, PAs are faced with increasing administrative responsibilities, particularly with the evolution of ACGME policies, the Next Accreditation System, and the 10 -year Self-Study process. ${ }^{13}$ PAs share the burden of these administrative tasks while often also serving as managers of and mentors for their residents and fellows. ${ }^{14}$ While the direct impact of PA burnout on trainee burnout is unknown, PAs are a critically important component of the training program team and often have a personal, professional, and very meaningful relationship with their residents, fellows, and faculty. Understanding that burnout can be "contagious," organizations should be encouraged to address burnout across the entire learning environment in a comprehensive, inclusive, and "unsiloed" approach. Understanding the prevalence of burnout among all in the field may help institutions better address the escalating physician burnout crisis.

With ACGME and institutional leadership adopting policies and interventions to better address the changing medical education climate, we sought to provide an in-depth look at the burnout of residency and fellowship PAs from across the country who work in varying specialties and assess whether levels change over the cadence of an academic year. In addition, we aimed to analyze the relationship between burnout and isolation, sleep quality, and perceived support on the job.

This longitudinal study stems from a baseline, crosssectional study and reports additional findings. ${ }^{15}$ Changes in burnout are examined over the course of an academic year and potential explanations for any changes are provided. Readers may find that the original study provides additional background information and context. ${ }^{15}$

\section{METHODS}

\section{Participant Recruitment}

A total of 931 eligible participants were recruited from a sample of 1084 individuals who expressed an interest in participating. We estimate that this represents a sampling of approximately $11 \%(1084 / 10,205)$ of the PA population in the USA (ThalamusGME, personal communication, April 19, 2019).

Participants were recruited for this voluntary study through multiple online PA specialty groups such as the Association of Pediatric Program Directors - Program Coordinators and the Association of Family Medicine Administration, and online forums that include the Association for Hospital Medical Education (AHME) and LinkedIn. PAs were deemed eligible to participate based on the scope of their role as a PA. The methods, including inclusion criteria, and recruitment of all study participants are described in greater detail elsewhere. ${ }^{15}$

\section{Data Collection}

In July 2017, a baseline survey was electronically disseminated to all individuals who voluntarily emailed the study team with an interest in participating. Following baseline data collection, quarterly surveys were sent to all remaining eligible participants over the rest (September 2017-June 2018) of the academic year. Only those who completed the baseline survey were eligible to complete the follow-up surveys. All participants were given 2 weeks to complete each quarterly survey.

The title "Program Administrators" was used to encourage all interested residency/fellowship coordinators, residency managers, etc. to participate and help encapsulate those whose job responsibilities fell under that of a program coordinator or another related job title. To ascertain continued eligibility status, participants were asked a series of questions at each data collection period to gauge whether they changed positions or had given notice to leave. If the participant responded that they had changed positions or had given notice to leave since the completion of the prior survey and their new position was not within the GME field, they were no longer eligible to participate, but were asked additional questions to determine the level of support received and overall job satisfaction in the position they had resigned from or had given notice to leave.

\section{Measurement Tools}

Burnout was measured using the Copenhagen Burnout Inventory $(\mathrm{CBI})$, a 19-item instrument used to assess personal, work-related, and client-related burnout in diverse clinical and non-clinical professions. ${ }^{16}$ Kristensen et al. defined client-related burnout as "The degree of physical and psychological fatigue and exhaustion that is perceived by the person as related to his/her work with clients." 16 Questions centered on individuals' interaction with clients: "Do you find it hard to work with clients?" and "Are you tired of working with clients?" ${ }^{16}$ In our study, "clients" referred to interns, residents, fellows, and medical students.

A higher burnout score indicates a higher degree of burnout (no cutoffs were established). ${ }^{16}$ Possible burnout scores for each subscale ranged from 0 to 100 , with " 0 " representing a response of "Never/almost never or To a very low degree" and " 100 " representing a response of "Always or To a very high degree."

In addition to measuring burnout, we used the PROMIS29 Profile v1.0 4a (Patient-Reported Outcome Measurement Information System, 4-item) to measure the quality of sleep at follow-up ${ }^{17}$ and collected demographic characteristics that would help elucidate potential contributors to higher burnout scores. The creators of the system found that a score of $\geq 10$ represents persons who are on average somewhat more chronically ill than the general US population ${ }^{17}$; thus, we defined poor sleep quality as having a score of $\geq 10$ and good sleep quality as having a score of $<$ 10. A clinical sample (those with at least one chronic 
illness) was initially used as the reference population to derive the raw mean score of 10 , which suggests that greater sleep disturbance may be present in healthy individuals scoring $\geq 10$. $^{18,19}$ The PROMIS sleep disturbance instrument has construct validity and reliability ${ }^{20}$ and may be used in the general population.

\section{Statistical Analyses}

The generalized estimating equations (GEE) linear regression model (primary analysis) was used to identify changes in personal, work-related, and client-related burnout scores over the academic year (July 2017-June 2018), with baseline (July 2017) burnout scores used as the reference. The Bonferroni test (a priori) was used to adjust for multiple comparisons. Ten pairwise comparisons were made.

A one-way ANOVA test (secondary analysis) was used to analyze the average burnout score at the end of the academic year among participants across response level for questions pertaining to quality of sleep and feelings of support. The test adjusted for age, education, and length of time in position. Least squares means \pm standard deviation (SD) was reported. Statistical significance across all models was defined as having a $p$ value $<0.05$.

SAS software version 9.4 (SAS Institute, Inc.) was used for all statistical analyses. Study data were collected and managed using REDCap electronic data capture tools, CTSI 1UL1TR001430. ${ }^{21}$

The study protocol was deemed exempt by the Boston University Institutional Review Board.

\section{RESULTS}

\section{Study Population}

This longitudinal study represented PAs from more than 35 specialties including Internal Medicine (there was no statistically significant difference in burnout scores among this group relative to PAs in other specialties). ${ }^{15}$ Baseline data have been previously reported and a more detailed description of participant characteristics has been reported elsewhere. ${ }^{15}$

\section{Changes in Burnout}

Using the GEE linear regression model to examine changes in personal, work-related, and client-related burnout from baseline (July 2017) through the academic year, we observed a 3.67 decrease $(p<0.001 ; 95 \%$ CI -5.77 to -1.58$)$ in personal burnout score in the 3rd quarter (March 2018 (Table 1)), and in the same quarter, a similarly lower, -3.03 , work-related burnout score relative to baseline. July, which marks the beginning of the academic year, was shown to have the highest levels of personal and work-related burnout.

Client-related burnout scores fluctuated over the year, with the 4th quarter (June 2018) representing the highest level of burnout (an increase of 1.47 from baseline; $p=0.626 ; 95 \% \mathrm{CI}$ -0.75 to 3.70$)$.

\section{Response Rate}

Of the 1084 individuals nationally who emailed the study team to be enrolled and the 42 eligible PAs at our local institution, 933/1126 (82.9\%) completed the baseline assessment in its

Table 1 Changes in Burnout Score Among National Residency/Fellowship Program Administrators over the Academic Year $(N=931)$

\begin{tabular}{|c|c|c|c|c|c|}
\hline \multirow[t]{2}{*}{ Burnout subscales* } & \multirow[t]{2}{*}{ Assessment Period } & \multirow[b]{2}{*}{$\mathbf{B}^{\dagger}$} & \multirow[b]{2}{*}{ SE } & \multirow[b]{2}{*}{$p$ value ${ }^{\ddagger}$} & \multirow[b]{2}{*}{$(95 \% \mathrm{CI})^{\ddagger}$} \\
\hline & & & & & \\
\hline \multirow[t]{6}{*}{ Personal } & Baseline $^{\S}$ & ref & ref & ref & \\
\hline & September 2017 & -1.16 & 0.71 & 1.000 & $(-3.15$ to 0.83$)$ \\
\hline & December 2017 & -1.36 & 0.69 & 0.478 & $(-3.29$ to 0.57$)$ \\
\hline & March 2018 & -3.67 & 0.75 & $<0.001$ & $(-5.77$ to -1.58$)$ \\
\hline & June 2018 & -2.80 & 0.75 & 0.002 & $(-4.90$ to -0.70$)$ \\
\hline & $P$ value & & & $<0.001$ & \\
\hline \multirow[t]{6}{*}{ Work-related } & Baseline $^{\S}$ & ref & ref & ref & \\
\hline & September 2017 & -0.63 & 0.64 & 1.000 & $(-2.42$ to 1.16$)$ \\
\hline & December 2017 & -1.88 & 0.69 & 0.067 & $(-3.82$ to 0.07$)$ \\
\hline & March 2018 & -3.03 & 0.70 & $<0.001$ & $(-5.01$ to -1.06$)$ \\
\hline & June 2018 & -1.17 & 0.75 & 1.000 & $(-3.27$ to 0.94$)$ \\
\hline & $\mathrm{P}$ value & & & $<0.001$ & \\
\hline \multirow[t]{6}{*}{ Client-related } & Baseline $^{\S}$ & ref & ref & ref & \\
\hline & September 2017 & -1.46 & 0.74 & 0.491 & ( -3.54 to 0.62$)$ \\
\hline & December 2017 & -1.30 & 0.77 & 0.924 & $(-3.48$ to 0.87$)$ \\
\hline & March 2018 & -0.12 & 0.79 & 1.000 & $(-2.32$ to 2.09$)$ \\
\hline & June 2018 & 1.47 & 0.79 & 0.626 & $(-0.75$ to 3.70$)$ \\
\hline & $\mathrm{P}$ value & & & $<0.001$ & \\
\hline
\end{tabular}

"Copenhagen Burnout Inventory (CBI): scores ranged from 0 to 100 within each subscale, with a score of "100" signifying an "Always or To a very high degree" response, and a score of "0" signifying a "Never/almost never or To a very low degree" response

${ }^{\dagger}$ Calculated using the Generalized Estimating Equations (GEE) linear regression model for evaluating the changes in burnout score over the course of the academic year

${ }^{7}$ Bonferroni test used to adjust for multiple comparisons

${ }^{8}$ Reference

Significant at the 0.05 level (2-tailed)

${ }^{\text {CClient referred to intern, resident, fellow, and medical student }}$ 
entirety (Appendix Fig. 1). Response rates at the first quarterly follow-up in September 2017 were maintained among those eligible (728/931 (78.2\%)). Though the exact total number of PAs across the country is unknown, it is estimated to be approximately 10,205 (ThalamusGME, personal communication, April 19, 2019). Thus, for the baseline survey, our 1084 participants represent an $11 \%$ response rate.

\section{Burnout, Support, and Sleep Quality}

Elevated levels of client-related burnout at the end of the academic year (June) prompted further exploration into possible associations between support and burnout at this time of the year. Therefore, using a one-way ANOVA test, we examined changes in personal, work-related, and client-related burnout scores in June by responses to various questions/statements including, "Are you given the proper tools, resources, and/or training to succeed at your job?" (Table 2). This cross-sectional examination was conducted at the conclusion of the study after evaluating changes in burnout over the year, which revealed elevated levels of client-related burnout and a statistically significant difference in personal burnout scores in June from baseline. The model adjusted for age, education, and length of time in position. Those who responded "yes" to being given the proper tools/resources to succeed at their job had lower personal $(44.9 \pm 20.9)$, work-related $(44.0 \pm 21.5)$, and client-related $(28.5 \pm 20.7)$ burnout scores relative to those who responded unfavorably (no); $p<0.001$ across all three burnout subscales. At the first quarterly follow-up period in September, participants were asked whether they received support or encouragement from their program or department to attend conferences/ training sessions related to their PA role. Among the 728 respondents, 451 (62\%) indicated they received financial support and encouragement to attend conferences/trainings.

The relationship between sleep quality and burnout was also assessed. The average personal burnout score among those who had a good quality of sleep was lower $(-21.2 \pm$ 1.7 SE, $p<0.001)$ than those with poor sleep quality. Similarly, differences in work-related $(-19.8 \pm 1.8 \mathrm{SE}, p<0.001)$ and client-related $(-11.6 \pm 1.8 \mathrm{SE}, p<0.001)$ burnout scores among those with good sleep quality in relation to poor sleep quality were evident.

\section{Resigned from Position}

Between the 1st quarter (September 2017) and 4th quarter (June 2018), 105 participants stated that they had changed positions or had given notice to leave their position. Some of the reasons for changing positions included a combination of the following: offered another position (58/105 (55\%)), poor management $(43 / 105(41 \%))$, too stressful (40/105 (38\%)), low pay $(39 / 105(37 \%))$, and no interest in the position $(5 / 105(5 \%))$. Other reasons included retirement and opportunities for further education.

Table 2 Burnout Scores Among Program Administrators Across Response Levels of Perceived Support and Isolation, June 2018, $N=602$ (Adjusted)

\begin{tabular}{|c|c|c|c|c|c|c|}
\hline & \multicolumn{2}{|c|}{ Personal burnout score* } & \multicolumn{2}{|c|}{$\begin{array}{l}\text { Work-related burnout } \\
\text { score* }\end{array}$} & \multicolumn{2}{|c|}{$\begin{array}{l}\text { Client-related burnout } \\
\text { score* }\end{array}$} \\
\hline & LS-means $\pm \mathbf{S D}^{\dagger}$ & $p$ values & LS-means \pm SD $^{\dagger}$ & $p$ values & LS-means $\pm \mathbf{S D}^{\dagger}$ & $p$ values \\
\hline \multicolumn{7}{|l|}{ Characteristics } \\
\hline Given the proper tools/resources to succeed at job & & $<0.001^{\ddagger}$ & & $<0.001^{\ddagger}$ & & $<0.001^{*}$ \\
\hline No & $65.9 \pm 18.4$ & & $68.3 \pm 19.4$ & & $41.3 \pm 23.4$ & \\
\hline Yes & $44.9 \pm 20.9$ & & $44.0 \pm 21.5$ & & $28.5 \pm 20.7$ & \\
\hline Feel appreciated and/or respected at institution & & $<0.001^{*}$ & & $<0.001^{*}$ & & $<0.001^{*}$ \\
\hline No & $62.4 \pm 19.7$ & & $64.7 \pm 20.2$ & & $40.7 \pm 22.7$ & \\
\hline Yes & $44.0 \pm 20.9$ & & $42.7 \pm 21.5$ & & $27.2 \pm 20.4$ & \\
\hline Isolated in current position & & $<0.001^{\ddagger}$ & & $<0.001^{*}$ & & $<0.001^{*}$ \\
\hline Strongly disagree & $35.0 \pm 19.9$ & & $31.6 \pm 21.0$ & & $21.3 \pm 18.8$ & \\
\hline Disagree & $45.4 \pm 19.2$ & & $45.1 \pm 19.8$ & & $27.7 \pm 20.9$ & \\
\hline Neutral & $52.1 \pm 20.6$ & & $52.9 \pm 20.3$ & & $34.3 \pm 21.9$ & \\
\hline Agree & $61.4 \pm 19.7$ & & $62.2 \pm 19.6$ & & $39.8 \pm 21.2$ & \\
\hline Strongly agree & $69.1 \pm 18.4$ & & $72.5 \pm 20.8$ & & $42.3 \pm 23.7$ & \\
\hline Contacted by program personnel & & $<0.001^{*}$ & & $<0.001^{\ddagger}$ & & $<0.001^{*}$ \\
\hline Never & $41.9 \pm 25.2$ & & $39.2 \pm 27.2$ & & $22.4 \pm 24.3$ & \\
\hline Rarely & $43.9 \pm 20.8$ & & $44.1 \pm 22.3$ & & $28.2 \pm 21.9$ & \\
\hline Sometimes & $52.0 \pm 20.7$ & & $52.2 \pm 21.9$ & & $32.4 \pm 20.8$ & \\
\hline Often & $54.8 \pm 20.7$ & & $54.6 \pm 21.7$ & & $37.6 \pm 19.8$ & \\
\hline Very often & $64.7 \pm 23.3$ & & $64.8 \pm 23.4$ & & $38.9 \pm 25.8$ & \\
\hline Sleep quality ${ }^{\S}$ & & $<0.001^{+}$ & & $<0.001^{\ddagger}$ & & $<0.001^{+}$ \\
\hline Poor & $57.8 \pm 20.0$ & & $57.2 \pm 21.4$ & & $35.9 \pm 22.3$ & \\
\hline Good & $36.6 \pm 19.4$ & & $37.4 \pm 21.6$ & & $24.3 \pm 19.7$ & \\
\hline
\end{tabular}

"Copenhagen Burnout Inventory (CBI): Scores ranged from 0 to 100 within each subscale, with a score of "100" signifying an "Always or To a very high degree" response, and a score of " 0 " signifying a "Never/almost never or To a very low degree" response

${ }^{\dagger}$ Calculated using one-way ANOVA and reported as least squares means (LS-means) \pm standard deviation (SD). Adjusted for age, education, and length of time in position

Significant at the 0.05 level (2-tailed)

SPROMIS-29: Patient Reported Outcome Measurement Information System. Per the literature, a score of $\geq 10$ represents persons who are on average somewhat more chronically ill than the general US population. We classified those with a score of $\geq 10$ as having poor sleep quality and those with a score of $<10$ as having good sleep quality 
Of the 105, 42 (40\%) stated that their new position was not in the GME field. Of the 42, 13 (31\%) did not feel like a valued asset to their program, while 19 of the 42 (45\%) expressed they were not given the proper tools, resources, and/or training to succeed at their job.

\section{DISCUSSION}

As depicted in Table 1, burnout levels among PAs fluctuated over the academic year. A possible explanation may be the shifts in responsibilities that occur during this time period. Personal and work-related burnout is most evident at the start of the academic year in July, with this month marking the busiest time of the year and the unofficial start of recruiting season. PAs represent the first point of contact for trainees, working psychosocially to not only establish a supportive learning and professional environment built on trust and collegiality but also administratively to reliably address the evolving array of new hire work-related questions as well as the timely completion of all required orientation and onboarding tasks. From August through January, PAs organize various aspects of the recruiting process that include managing and reviewing incoming applications. ${ }^{22}$ In November and December, programs are responsible for holding clinical competency committees, completing milestones, and scheduling semi-annual reviews with all trainees. For many programs, in-training exams are typically ordered and proctored during this time.

By February, most residency programs have completed recruitment, potentially leaving PAs with more downtime than at any other time in the year. As our results show, some PAs have the opportunity for professional development and are allotted funds to travel to national conferences. This active integration into a broader community may potentially represent a protective factor that mitigates feelings of isolation and lessens burnout. At the end of the academic year in June, program administrators are faced with additional trainees as they onboard new learners and graduate senior residents and fellows, which may contribute to the higher levels of clientrelated burnout during this period.

In addition to our study's findings on isolation and perceived support (Table 2), findings from prior studies have suggested that social and geographical isolation greatly impact burnout. ${ }^{23,24}$ Isolation and loneliness play a significant role in the well-being of clinical and administrative staff. A study examining the link between loneliness and burnout in US residents, in which loneliness was defined as the perceived lack of connection to others, demonstrated that higher burnout resulted in higher loneliness scores. ${ }^{25}$ The literature further highlights the impact a caustic work environment has on an individual's organizational commitment, as lonelier staff feel less emotionally and socially connected to colleagues, regardless of position title. ${ }^{26,27}$ Furthermore, studies have found that those working in healthcare with higher levels of burnout also had poorer sleep quality, factors that correlate with job performance. $^{28,29}$ Our study findings support the link between sleep and burnout.

While this is the largest known national longitudinal study that examines burnout among residency and fellowship coordinators across specialties, there are some important limitations. Though the demographic characteristics of PAs across the USA are unknown, our study group was disproportionately female (97\%), a group that may or may not be more susceptible to work-related burnout than their male counterparts. Women may be more open to reporting their sentiments. ${ }^{30}$ It is also reported that men and women respond differently to work and non-work related stressors. ${ }^{31}$ Nevertheless, the gender breakdown of our local PA population mirrors the breakdown of our entire national participant group, suggesting generalizability.

Participation in this study was voluntary. Most importantly, particularly given the size of many Internal Medicine training programs, $10 \%$ of our national study group worked in an Internal Medicine Residency Program at data collection, the most represented specialty in our study. Though specialty forums were used to recruit participants, selection bias may have been introduced, where those who perceived themselves to be burned out were more inclined to participate. While retrospective data on burnout levels of participants, as well as burnout data from participants who left their position and were no longer eligible to participate, were not obtained, our study group's burnout scores do mirror the scores of those who work clinically in diverse geographical settings. ${ }^{32,33}$ To our knowledge, there is no known measure of "educational" or "clinical" significance of a statistical change in burnout score of $<5$. Lastly, 270 subjects were lost to follow-up at the conclusion of the study. These subjects were still eligible to participate and did not opt out at any period during the study.

Burnout rates vary over the academic year and may be impacted by feelings of isolation and levels of perceived support. Feeling appreciated and/or respected at one's institution and being given the proper tools/resources to succeed are shown to positively impact burnout scores, corroborating the notion that burnout may be influenced by a supportive and resource enriched working environment. Appreciating that burnout among PAs changes over the course of the academic year may provide important opportunities for organizations to tailor offerings such as professional development courses, support systems, and intentional frameworks to address the cyclical nature of the position's pressures and cadence. Furthermore, a more proactive approach to address sentiments of feeling undervalued may also encourage a better work/life balance and foster a stronger PA-trainee connection. Creating an overall, more positive, engaged, and supportive work environment leading to an empowered workforce could potentially reduce staff turnover and improve morale. With the goal of reducing burnout more broadly in medical education, future studies should focus on critically assessing what tools may be implemented throughout the year to reliably reduce the effect of burnout among PAs across specialties. 
Corresponding Author: Jeffrey I. Schneider, MD; Department of Emergency Medicine, Boston University School of Medicine, Boston, MA, USA (e-mail: jeffrey.schneider@bmc.org).

Funding information This publication was supported by the National Center for Advancing Translational Sciences, National Institutes of Health, through BU-CTSI Grant Number 1UL1TROO1430.

\section{Compliance with Ethical Standards:}

The study protocol was deemed exempt by the Boston University Institutional Review Board.

Conflict of Interest: The authors declare that they do not have a conflict of interest.

Disclaimer: The study contents are solely the responsibility of the authors and do not necessarily represent the official views of the NIH.

\section{REFERENCES}

1. Maslach C, Jackson SE. The measurement of experienced burnout. J Occup Behav 1981; 2(2):99-113.

2. Shanafelt TD, Hasan O, Dyrbye LN, et al. Changes in burnout and satisfaction with work-life balance in physicians and the general US working population between 2011 and 2014. Mayo Clin Proc 2015;90(12): 1600-1613.

3. Ewen AM, Gardiner PM, Palma S, Whitley K, Schneider JI. We matter too! Addressing the wellness of program coordinators in graduate medical education. J Contin Educ Heal Prof 2018;38(3):165-170.

4. Ofei-Dodoo S, Scripter C, Kellerman R, Haynes C, Marquise M, Bachman C. Burnout and job satisfaction among family medicine residency coordinators: results from a national survey. Fam Med 2018;50(9):679-684.

5. Bakker AB, Le Blanc PM, Schaufeli WB. Burnout contagion among intensive care nurses. J Adv Nurs 2005;51(3):276-287.

6. Welp A, Meier LL, Manser T. The interplay between teamwork, clinicians emotional exhaustion, and clinician-rated patient safety: a longitudinal study. Crit Care 2016;20(1):110.

7. Siedsma M, Emlet L. Physician burnout: can we make a difference together? Crit Care. 2015;19(273).

8. Poonja Z, O'Brien P, Cross E, et al. Sleep and exercise in emergency medicine residents: an observational pilot study exploring the utility of wearable activity monitors for monitoring wellness. Cureus. 2018;10(7):e2973.

9. Spiotta AM, Fargen KM, Patel S, Larrew T, Turner RD. Impact of a residency-integrated wellness program on resident mental health, sleepiness, and quality of life. Neurosurgery. 2018;0:1-6.

10. Callahan K, Christman G, Maltby L. Battling burnout: strategies for promoting physician wellness. Adv Pediatr Infect Dis 2018;65(1):1-17.

11. Wiederhold BK, Cipresso P, Pizzioli D, Wiederhold M, Riva G. Intervention for physician burnout: a systematic review. Open Med 2018; 13:253-263.

12. Accreditation Council for Graduate Medical Education. ACGME Coordinator Advisory Group Charge. https://www.acgme.org/Program-Direc tors-and-Coordinators/ACGME-Coordinator-Advisory-Group/ACGMECoordinator-Advisory-Group-Charge. Accessed November 7, 2018.

13. Accreditation Council for Graduate Medical Education. Common program requirements (residency). http://www.acgme.org/Portals/0/PFAssets/ProgramRequirements/CPRResidency2019.pdf. Accessed
November 7, 2018.

14. Stuckelman J, Zavatchen SE, Jones SA. The evolving role of the program coordinator: five essential skills for the coordinator toolbox. Acad Radiol 2017;24(6):725-729.

15. Ewen AM, Higgins CSSM, Palma S, Whitley K, Schneider JI. Residency and fellowship program administrator burnout: measuring its magnitude. J Grad Med Educ 2019;11(4):402-409.

16. Kristensen TS, Borritz M, Villadsen $\mathbf{E}$, Christensen KB. The copenhagen burnout inventory: a new tool for the assessment of burnout. Work Stress 2005;19(3): 192-207.

17. PROMIS-29 Profile v1.0. 2008-2012 PROMIS Health Organization and PROMIS Cooperative Group. https://www.assessmentcenter.net/documents/PROMIS\%20Profile\%20Scoring\%20Manual.pdf. Accessed February 7, 2017.

18. PROMIS Adult Profile Instruments. 2020 Patient-Reported Outcomes Measurement Information System. http://www.healthmeasures.net/ images/PROMIS/manuals/PROMIS_Adult_Profile_Scoring_Manual.pdf. Accessed February 19, 2020.

19. HealthMeasures. PROMIS $®$ Reference Populations. http://www.healthmeasures.net/score-and-interpret/interpret-scores/promis/referencepopulations. Accessed February 19, 2020

20. Buysse DJ, Yu L, Moul DE, et al. Development and validation of patientreported outcome measures for sleep disturbance and sleep-related impairments. Sleep. 2010;33(6):781-792.

21. Harris PA, Taylor R, Thielke R, Payne J, Gonzalez N, Conde JG. Research electronic data capture (REDCap) - A metadata-driven methodology and workflow process for providing translational research informatics support. J Biomed Inform 2009;42(2):377-381.

22. Otterstad D. The role of the residency coordinator. Acad Radiol 2003; 10(Suppl 1):S48-S53.

23. Truchot D, Andela M. Burnout and hopelessness among farmers: the farmers stressors inventory. Soc Psychiatry Psychiatr Epidemiol 2018;53(8):859-867.

24. Kase SM, Waldman ED, Weintraub AS. A cross-sectional pilot study of compassion fatigue, burnout, and compassion satisfaction in pediatric palliative care providers in the United States. Palliat Support Care 2018;5:1-7.

25. Shapiro J, Zhang B, Warm EJ. Residency as a social network: burnout, loneliness, and social network centrality. J Grad Med Educ 2015;7(4):617-623.

26. Holt-Lunstad J. Fostering social connection in the workplace. Am J Health Promot 2018;32(5): 1307-1312.

27. Bauer S, Silver $\mathbf{L}$. The impact of job isolation on new principals' sense of efficacy, job satisfaction, burnout and persistence. J Educ Adm 2018;56(3):315-331.

28. Wolf MR, Rosenstock JB. Inadequate sleep and exercise associated with burnout and depression among medical students. Acad Psychiatry 2017;41(2): 174-179.

29. Chin W, Guo YL, Hung YJ, Yang CY, Shiao JS. Short sleep duration is dose-dependently related to job strain and burnout in nurses: a cross sectional survey. Int J Nurs Stud 2015;52(1):297-306.

30. Baumeister RF, Bushman BJ. Social Psychology and Human Nature 5e. Boston: Cengage; 2019:218.

31. Cinamon RG, Rich Y. Gender differences in the importance of work and family roles: implications for work-family conflict. Sex Roles 2002;47:531-541.

32. Ratnakaran B, Prabhakaran A, Karunakaran V. Prevalence of burnout and its correlates among residents in a tertiary medical center in Kerala, India: a cross-sectional study. J Postgrad Med 2016;62(3):157-161.

33. Kassam, A., Horton, J., Shoimer, I., Patten, S. Predictors of Well-Being in Resident Physicians: A Descriptive and Psychometric Study. J Graduate Med Educ 2015;7:70-74.

Publisher's Note: Springer Nature remains neutral with regard to jurisdictional claims in published maps and institutional affiliations. 